\title{
Flagellin Alleviates Airway Allergic Response by Stabilizing Eosinophils through Modulating Oxidative Stress
}

\author{
Xiang-Qian Luo ${ }^{a}$ Jun Liu ${ }^{b}$ Li-Hua Moa,c Gui Yang ${ }^{d}$ Fei Ma ${ }^{e}$ Yan Ning ${ }^{e}$ \\ Ping-Chang Yang ${ }^{c}$ Da-Bo Liu ${ }^{a}$ \\ aDepartment of Pediatric Otolaryngology, Shenzhen Hospital, Southern Medical University, Shenzhen, China; \\ bDepartment of Medical Records Management, Shenzhen Maternal \& Child Healthcare Hospital, \\ Shenzhen, China; 'CGuangdong Provincial Key Laboratory of Regional Immunity and Diseases, and \\ Research Center of Allergy \& Immunology, Shenzhen University School of Medicine, Shenzhen, China; \\ ${ }^{\mathrm{d}}$ Department of Otolaryngology, Longgang Central Hospital, Shenzhen, China; 'Department of Traditional \\ Chinese Medicine, Shenzhen Maternity \& Child Healthcare Hospital, Shenzhen, China
}

\section{Keywords}

Eosinophil · Flagellin · Toll-like receptor-5 · Allergy ·

Oxidative stress

\begin{abstract}
Eosinophil (Eo) degranulation plays a central role in the initiations of allergic attacks. Flagellin (FGN), the major component of bacterial flagella, has immune regulatory functions. This study aims to investigate the role of FGN in alleviating the allergic reaction by stabilizing Eos. A toll-like receptor 5-knockout mouse strain was employed to test the role of FGN in stabilizing Eos. An airway allergy mouse model was developed to test the administration of FGN in alleviating the airway allergy by stabilizing Eos. The results showed that FGN was required in stabilizing Eos in the airway tissues. FGN prevented specific antigen-induced Eo activation. Oxidative stress was associated with the antigen-induced Eo activation that could be counteracted by the presence of FGN. The FGN levels were lower and chymase levels were higher in the airway tissues of mice with allergic inflammation. Negative correlation was detected between the data of FGN and chymase
\end{abstract}

in the lung tissues. Chymase physically contacted FGN to speed up its degradation. The administration of FGN alleviated experimental allergic inflammation in the mouse airways by stabilized Eos in the lung tissues. In conclusion, FGN contributes to Eo stabilization. The administration of FGN alleviates the experimental airway allergy. The data suggest that FGN can be a candidate to be employed in the treatment of allergic disorders.

(c) 2021 The Author(s)

Published by S. Karger AG, Basel

\section{Introduction}

Eosinophils (Eos) are a fraction of the major effector cells in allergic diseases, most inflammatory diseases, and some tumors [1]. An anatomical feature of Eos is that the cytoplasm contains numerous granules, which consist of many chemical substances; some of them are inflammatory mediators, for example, major basic protein (MBP),

Xiang-Qian Luo, Jun Liu, and Li-Hua Mo contributed equally to this work.

(C) 2021 The Author(s)

Published by S. Karger AG, Basel

This is an Open Access article licensed under the Creative Commons Attribution-NonCommercial-4.0 International License (CC BY-NC) (http://www.karger.com/Services/OpenAccessLicense), applicable to the online version of the article only. Usage and distribution for commercial purposes requires written permission.
Correspondence to:

Ping-Chang Yang, pcy2356@ szu.edu.cn

Da-Bo Liu, liudb@126.com 
Eo cationic protein, and Eo peroxidase (EPX) [2]. Upon activation, Eos degranulate and release chemical mediators that induce inflammatory reactions in the local tissues [3]. Although the research in Eo activation has advanced greatly in the recent years, the underlying mechanism by which Eos maintaining the stabilization status remains incompletely understood.

Another fraction of effector cell in allergic responses is mast cells. The mast cells also contain many granules that contain many inflammatory mediators, such as histamine, chymases, serotonin, and leukotrienes. Upon activation, the mast cells release these mediators to evoke allergic attacks [4]. The chymases are proteinases that can degrade other proteins [5]. The mast cells also produce eotaxin that attracts Eos to the lesion sites [6]. Yet, it is lesser understood what is the interaction between the mast cells and Eos.

It is reported that Eos can be activated by multiple factors, including antigen/antibody $(\mathrm{Ab})$ interaction, exposure to microbial stimulation, temperature changes, and mechanic forces [3]. Eos naturally express Fc $\gamma$ RII and Fc $\gamma$ RIII, but express low or undetectable Fc $\gamma$ RI at the steady status [7], the latter may be regulated upon activation [8-10]. However, these stimulatory factors generally exist in the human body, why Eos are not activated under physiological conditions is incompletely understood. Specifically, the IgG Abs generally exist in the body and have chances to contact the Fc $\gamma$ Rs on the surface of Eos [9]; how Eos maintain the stabilization status in a healthy condition also remains to be investigated.

Eos express toll-like receptor-5 (TLR5); exposure to TLR5 ligand and flagellin (FGN) can induce Eos to express and release inflammatory cytokines, such as IL-1 $\beta$, IL-6, and IL-8, but not cause Eo degranulation [11]. FGN is the major part of flagellum, the locomotory organ of Gram-negative bacteria. The published data indicate that, not only associated with the bacterial virulence, FGN also has immune regulatory functions [12]. Repeated injection with FGN can induce the production of FGN-specific IgA that alters microbiota composition, reduces fecal FGN amounts and suppresses inflammation in the colon [13, 14]. Although FGN seems to involve the development of allergic disorders [15], it seems involving the regulation of Eo infiltration [16]. Thus, the role of FGN in the regulation of Eo activities needs to be further investigated.

Based on the information above, we hypothesize that FGN contributes to the Eo stabilization. In this study, we observed that lacking the TLR5 signals resulted in the spontaneous Eo activation in the airway tissues. Administration of FGN alleviated experimental airway allergy by stabilizing Eos in the airway tissues.

\section{Materials and Methods}

\section{Reagents}

Abs of TLR5 (Cat\#: H-11), MBP (F-6), Fc $\gamma$ RI (C-6), Fc $\gamma$ RII (B-4), Fc $\gamma$ RIII (ASH 1975), CD69 (D-3), SOD (B-1), chymase (CC1), FGN (6,321), ubiquitin (A-5), CD11c (B-6; Alexa Fluor ${ }^{\circledR}$ 488), TLR5 (H-11; Alexa Fluor $\left.{ }^{\circledR} 546\right)$, Siglec-F (F-7; Alexa Fluor ${ }^{\circledR}$ 594), MBP (F-6; Alexa Fluor ${ }^{\circledR}$ 647), CD45 (35-Z6; Alexa Fluor ${ }^{\circledR}$ 790), and CD69 (D-3; Alexa Fluor ${ }^{\circledR}$ 546) were purchased from Santa Cruz Biotech (Santa Cruz, CA, USA). CellROX Deep Red Reagent, and enzyme-linked immunosorbent assay (ELISA) kits of MBP, EPX, CD69, FGN, chymase, IL-4, IL-5, IL-13, and sCD69 were purchased from Dakewe Biomart (Beijing, China). Ovalbumin (OVA)-specific IgE and IgG ELISA kit were purchased from Biocon Inc (Shanghai, China). FGN and mouse IgG protein were purchased from Abcam (Cambridge, MA, USA). Recombinant chymase was purchased from Shanghai Lianmai Biotech (Shanghai, China). Materials and reagents for real-time quantitative PCR, Western blotting, and immunoprecipitation (IP) were purchased from Invitrogen (Carlsbad, CA, USA).

Mice

C57BL/6 mice, 8- to 10 -week old, were purchased from the Guangdong Experimental Animal Center (Guangdong, China). TLR5 knockout (KO) mice were purchased from Jackson Laboratory (Bar Harbor, ME, USA). The mice were maintained in a specific pathogen-free facility with access to water and food freely. The animal experimental procedures were approved by the Animal EthicalCommitteeatSouthern MedicalUniversity(SMUAEC2019022).

\section{Development of an Airway Allergy Mouse Model}

Mice carrying TLR5-deficient Eos (KO mice) and C57BL/6 mice (wild type $[\mathrm{WT}]$ ) were subcutaneously injected with OVA $(0.1 \mathrm{mg} /$ mouse) mixed in $0.1 \mathrm{~mL}$ alum on the back skin on days 0 and 7 , respectively. The mice were boosted by treating with OVA $(5 \mathrm{mg} / \mathrm{mL})$ in nasal instillation (50 uL per nostril) daily between day 15 and day 21 . Airway responsiveness to methacholine challenge was assessed on day 22. On day 23 , the mice were challenged by nasal instillation with OVA $(5 \mathrm{mg} / \mathrm{mL} ; 50 \mu \mathrm{L}$ per nostril). Two hours later, the mice were sacrificed by recapitalization; blood samples were collected for further experiments.

\section{Enzyme-Linked Immunosorbent Assay}

The levels of OVA-specific IgE (Biocon Inc., Shanghai, China), OVA-specific IgG (Biocon Inc., Shanghai, China), and cytokines in the serum or culture supernatant were determined by ELISA with commercial reagent kits following the manufacturer's instructions.

\section{Treatment of Mice with FGN}

The mice were treated with FGN $(1 \mathrm{mg} / \mathrm{mL})$ by nasal instillation at $50 \mu \mathrm{L} /$ nostril daily in parallel with the antigen boosting (days 15-21) in the course of the airway allergy development.

\section{Assessment of Mouse Airway Responsiveness}

The mice were challenged by inhalation of methacholine at gradient concentrations. The airway resistance was recorded by the FinePointe ${ }^{\mathrm{TM}}$ Noninvasive Airway Mechanics system (Buxco Electronics, Inc., Wilmington, NC, USA) and used as indicators of the airway responsiveness. 
Collection of Bronchoalveolar Lavage Fluids

Immediately after the sacrifice, the trachea was opened. Warm $\left(37^{\circ} \mathrm{C}\right)$ saline $(1 \mathrm{~mL})$ was instilled into the lungs with a $1-\mathrm{mL}$ syringe; the fluids were then collected with the same syringe. The lavage was repeated 3 times and bronchoalveolar lavage fluids (BALFs) collected each time was pooled. BALF of 3 times was pooled and analyzed in further experiments.

\section{Histology}

A piece of the middle right lobe lung tissue was excised, fixed with $4 \%$ formalin overnight, and processed for paraffin sections. The sections were stained with hematoxylin and eosin. The lung histology was observed under a light microscope.

\section{Assessment of the Role of TLR5 Signaling in the Eo}

Stabilization

TLR5 KO mice and WT mice were sacrificed. Blood samples were collected. The serum was isolated from the blood samples. The levels of MBP and EPX in the serum and BALF were determined by ELISA and used as indicators of Eo activation.

\section{Eo Purification}

The lungs were excised upon the sacrifice and cut into small pieces (about $2 \times 2 \times 2 \mathrm{~mm}$ in size). The tissues were incubated with collagenase IV $(1 \mathrm{mg} / \mathrm{mL})$ at $37^{\circ} \mathrm{C}$ for $20 \mathrm{~min}$ with mild agitation. Lysates were filtered through a cell strainer $(70 \mu \mathrm{m}$ first and then $40 \mu \mathrm{m}$ ). Single cells were collected by centrifugation at $500 \mathrm{~g}$ for $5 \mathrm{~min}$. After washing with PBS 3 times, the single cells were stained with FITC-CD45 Ab, PE-Siglec-F Ab, and APC-CD11c $\mathrm{Ab}$. The cells were analyzed by flow cytometry (FCS; BD Aria). $\mathrm{CD} 45^{+}$cells were gated first, and the Siglec-F $\mathrm{F}^{+} \mathrm{CD} 11 \mathrm{c}^{+}$cells were then sorted from the $\mathrm{CD} 45^{+}$cells to be used as Eos. To isolate the activated Eos, $\mathrm{CD}^{+} 9^{+}$(an activation marker) Siglec- $\mathrm{F}^{+} \mathrm{CD} 11 \mathrm{c}^{+}$ cells were sorted. As checked by FCS, the purity of isolated Eos was $>96 \%$; the viability of isolated Eos was $>99 \%$.

\section{Eo Culture}

Isolated Eos were cultured in RPMI1640 medium supplemented with $10 \%$ fetal calf serum, $100 \mathrm{U} / \mathrm{mL}$ penicillin, $0.1 \mathrm{mg} / \mathrm{mL}$ streptomycin, $2 \mathrm{~mm}$ glutamine, and $10 \mathrm{ng} / \mathrm{mL}$ IL-5. The viability of Eos was $>99 \%$ as assessed by the trypan blue exclusion assay.

\section{Flow Cytometry}

On the surface staining, the cells were stained with fluorescence-labeled primary Abs (Ab types are denoted in relevant figures) or isotype IgG diluted in 1:100 for $30 \mathrm{~min}$ at $4^{\circ} \mathrm{C}$. After washing with PBS 3 times, cells were analyzed with a flow cytometer (FACSCanto II, BD Bioscience). In the intracellular staining, the cells were fixed with $1 \%$ paraformaldehyde (containing $0.05 \%$ Triton X-100 to enhance the cell membrane permeability) for $1 \mathrm{~h}$. The rest of the procedures were the same as that of surface staining. The data were analyzed with FlowJo (TreeStar Inc., Ashland, OR, USA). The data obtained from the isotype IgG staining were used as the gating references.

\section{Protein Extracts}

Cells were collected from the relevant experiments and incubated with a lysis buffer (1.5 mM $\mathrm{MgCl}_{2}, 10 \mathrm{mM}$ HEPES, $0.5 \mathrm{mM}$ DTT, $10 \mathrm{~mm} \mathrm{KCl}, 0.05 \%$ NP40, $1 \mathrm{mM}$ EDTA, and protease inhibitor cocktail). Lysates were centrifuged at $13,000 \mathrm{~g}$ for $10 \mathrm{~min}$. The supernatant was collected and used as cytosolic proteins. The procedures were performed at $4^{\circ} \mathrm{C}$.

\section{Western Blotting}

Proteins were separated by SDS-PAGE and transferred onto a PVDF membrane. After incubating with $5 \%$ skim milk for 30 min to block nonspecific binding, the membrane was incubated with the primary Abs (diluted in 1:300-500), washed with Tris-buffered saline (TBST, containing $0.05 \%$ Tween 20 ), incubated with secondary Abs (conjugated with horseradish peroxidase; diluted in 1:5,000), and washed 3 times. Immunoblots on the membrane were developed with enhanced chemiluminescence and photographed in an imaging device (UVP, Cambridge, UK).

\section{Immunoprecipitation}

To detect protein complexes, IP was employed. Proteins were extracted from the relevant cells collected from experiments. Protein $\mathrm{G}$ agarose beads were added to the proteins and incubated for $1 \mathrm{~h}$ to adsorb preexisting immune complexes. The beads were removed by centrifugation at $5,000 \mathrm{~g}$ for $10 \mathrm{~min}$. The supernatant was collected and incubated with Abs of interest at $1 \mathrm{ug} / \mathrm{mL}$ or isotype IgG for $2 \mathrm{~h}$ to form immune complexes. Protein $\mathrm{G}$ agarose beads were then added to the samples to precipitate the immune complexes. After centrifuging at 13,000 $\mathrm{g}$ for $10 \mathrm{~min}$, the beads were collected; proteins on beads were eluted with an eluting buffer and analyzed by Western blotting. The membranes were blotted using Abs as denoted in relevant figures. The input was used as a control.

\section{ROS Detection in Eos by Flow Cytometry}

We used CellROX Deep Red Reagent to detect intracellular reactive oxygen species (ROS) in Eos, which freely diffuses into the cells to be oxidized by ROS and becomes highly fluorescent. Eos were incubated with $1 \mu \mathrm{M}$ CellROX Deep Red Reagent at $37^{\circ} \mathrm{C}$ for $30 \mathrm{~min}$, and the cells were analyzed by FCS.

\section{SOD Real-Time Quantitative PCR}

Total RNA was extracted from Eos with the TRIzol reagents and converted to $\mathrm{cDNA}$ with a reverse transcription reagent kit following the manufacturer's instructions. Samples were amplified in a qPCR device (CFX96; Bio-Rad) with the SYBR Green Super Mix and the presence of SOD primers (AACCATCCACTTCGAGCAGA and GGTCTCCAACATGCCTCTCT). The results were calculated with the $2-\Delta \Delta \mathrm{Ct}$ method against the housekeeping gene $\beta$-actin and presented as the relative quantification.

\section{Assessment of FGN Degradation by Chymase}

Recombinant chymase $(0,0.001$ and $0.01,0.1 \mu \mathrm{g} / \mathrm{mL}$, respectively) was mixed with FGN $(1 \mu \mathrm{g} / \mathrm{mL})$. The mixture was incubated at $37^{\circ} \mathrm{C}$ for $30 \mathrm{~min}$. The FGN levels in the samples were assessed by ELISA.

\section{Statistics}

Each sample was analyzed in triplicate; the average of thee readouts was used as 1 datum. Each experiment was repeated at least 3 times. The data are presented as mean \pm SEM. The difference between 2 groups was determined by the Student $t$-test. ANOVA followed by Dunnett's test or Bonferroni test was performed for the multiple comparisons. A $p<0.05$ was considered statistically significant. 



Fig. 1. Serum Eo mediator levels are higher in TLR5 KO mice. a Single cells were isolated from the lung tissues and analyzed by FCS. Gated FCS plots show TLR5 ${ }^{+}$Eos. b, c Eos were isolated by FCS (b); isolated Eos show $\mathrm{MBP}^{+}(\mathbf{c})$. d FCS histograms show KO Eos do not express TLR5. e Immunoblots show TLR5 protein levels in Eos. $\mathbf{f}-\mathbf{h}$ Bars show serum MBP (f) or BALF MBP $(\mathbf{g})$ or serum sCD69 (h) levels. i, $\mathbf{j}$ Eos were isolated from naive WT mice and cultured in the presence or absence of FGN $(100 \mathrm{ng} / \mathrm{mL})$ for $48 \mathrm{~h}$. Bars indicate the levels of MBP (i) and sCD69 (j) in the cul- ture supernatant. The data of bars are presented as mean \pm SEM. ${ }^{*} p<0.01$ (the Student $t$ test), compared with the WT group (f-h) or the saline group $(\mathbf{i}, \mathbf{j})$. Each group consists of 6 mice. The data of a-e are from 1 experiment that represents 6 independent experiments. Each dot in bars presents data obtained from 1 sample. $\mathrm{EO}$, eosinophil; WT, wild type; KO, knockout; MBP, major basic protein; TLR5, toll-like receptor 5; BALF, bronchoalveolar lavage fluids; FGN, flagellin; FCS, flow cytometry. 




Fig. 2. Assessment of the role of FGN in stabilization of sensitized Eos. Eos were isolated from the WT and KO mouse airway tissues by FCS. a Gel graphs show the isolated Eos express MBP, Fc $\gamma$ RI, Fc $\gamma$ RII, and Fc $\gamma$ RIII. b-e Eos were sensitized to OVA-specific IgG and treated in the culture as denoted below the $x$ axis. b, c Bars indicate the levels of MBP and EPX in the culture supernatant. d-f CD69 expression in Eos. The data of bars are presented as mean \pm SEM of MBP (b) and EPX (c) in the culture from 3 experiments. ${ }^{*} p<0.05$ (ANOVA followed by Dunnett's test) compared with the WT group. OVA: OVA in the culture $(5 \mu \mathrm{g} / \mathrm{mL})$.

\section{Results}

\section{Flagellin Is Required in Maintaining Eo Stabilization}

WT mice and TLR5-(KO mice were employed in the present study. The single cells were isolated from the lung tissues and analyzed by FCS. The lung-isolated Eos expressed TLR5 that was not detected in TLR5 KO mice (Fig. 1a). Eos were purified by FCS (Fig. 1b-c). The pro-
BSA: BSA in the culture $(5 \mu \mathrm{g} / \mathrm{mL})$. FGN: FGN in the culture $(5 \mu \mathrm{g} /$ $\mathrm{mL})$. \# and $\mathrm{KO}$, Eos were isolated from TLR5 KO mice. The data of $\mathbf{a}$, e are from 1 experiment that represents 3 independent experiments. Each dot in bars presents data obtained from 1 sample. EO, eosinophil; WT, wild type; KO, knockout; MBP, major basic protein; TLR5, toll-like receptor 5; BALF, bronchoalveolar lavage fluid; FGN, flagellin; FCS, flow cytometry; OVA, ovalbumin; RQ, relative quantification; EPX, eosinophil peroxidases; MFI, median fluorescence intensity.

teins extracted from purified Eos (Fig. 1b-c) showed TLR5 proteins in WT Eos; TLR5 KO Eos did not have any TLR5 expression as shown by FCS and Western blotting assay (Fig. 1d-e). We found that the representative Eo mediator, $\mathrm{MBP}$, amounts were significantly increased in the serum as well as in BALF of $\mathrm{KO}$ mice as compared with WT mice (Fig. 1f-g). Prompted by the published data that the CD69 expression in the Eos is 
higher in patients with asthma $[17,18]$, we assessed the sCD69 levels in the serum samples. The results showed that the sCD69 levels in the serum were significantly higher in KO mice than that in WT mice (Fig. 1h). As TLR5 is the receptor of FGN, the results implicate that FGN may play a crucial role in the maintenance of Eo stabilization. To verify the results, Eos were isolated from WT mice by FCS and cultured in the presence or absence of FGN. The results showed that the spontaneous Eo activation was observed in the absence of FGN that could be prevented by the presence of FGN in the culture (Fig. 1i-j).

FGN Prevents Specific Antigen-Induced Eo Activation The data of Figure 1 suggest that FGN may contribute to Eo stabilization. We next investigated if FGN played a role in the prevention of the specific antigen-induced Eo activation. Following established procedures [19], Eos were isolated from the naive mice with Siglec-F and CD11c as the Eo surface markers [19]. The expression of MBP, Fc $\gamma$ RI, Fc $\gamma$ RII, and Fc $\gamma$ RIII was detected in isolated Eos (Fig. 2a). The previous reports indicate that specific antigen/IgG complexes bind Fc $\gamma$ RI or Fc $\gamma$ RIII on the Eo surface to sensitize and activate Eos $[9,20]$. Thus, Eos were sensitized by exposing to anti-OVA IgG in the culture overnight. The sensitized Eos were exposed to OVA in the culture in the presence or absence of FGN. We observed that exposure to the OVA, but not BSA (an irrelevant antigen), markedly activated Eos manifesting the release of MBP and EPX into the culture supernatant; the presence of FGN efficiently prevented the OVA-induced Eo activation that was abolished by the TLR 5 depletion (Fig. $2 \mathrm{~b}-\mathrm{c}$ ). We also observed that exposure to the specific antigen, OVA, markedly increased the CD69 expression in Eos (Fig. 2d-f). The results verify the effects of FGN on stabilizing Eos.

\section{Oxidative Stress Is Associated with the Antigen- Induced Eo Activation That Can Be Counteracted by FGN}

The published data indicate that the Eo activation results in oxidative stress [21], whether the oxidative species are increased inside Eos in response to exposing specific antigens remain to be elucidated. Prompted by the fact of exposing to specific antigens can activate sensitized Eos [9], we developed an asthma mouse model; the mice were challenged with nasal instillation containing the specific antigen, OVA, together with or without FGN in the course of sensitization. After the sacrifice, the single cells were isolated from the lung tissues and analyzed by FCS. The results showed marked Eo infiltration in the lung tissues of the asthma mice; the Eo counts were not reduced in the lungs by the administration of FGN (Fig. 3a-b). The OVA challenge significantly increased the levels of ROS and CD69 in Eos isolated from the lungs, which was prevented by FGN administration (Fig. 3c-f). We then isolated CD69 ${ }^{+}$Eos (CD69 expression is a sign of Eo activation [17]) from the lung tissuederived single cells to be analyzed by real-time quantitative PCR and Western blotting. The results showed that the SOD expression was markedly lower in antigen-activated Eos than naive control (NC) Eos, which was counteracted by the presence of FGN (Fig. 3g-h). We also found high MBP levels in BALF (Fig. 3i) that were positively correlated with the activated $\left(\mathrm{CD} 69^{+}\right)$Eos (Fig. 3j) and negatively correlated with SOD expression in the activated Eos (Fig. 3k). The results demonstrate that exposure to specific antigens increases oxidative stress in Eos that in turn activates Eos. Although the administration of FGN does not reduce the Eo infiltration in the asthma mice, it can counteract the oxidative stress to stabilize Eos.

FGN Amounts Are Lower in the Airway Tissues of Mice with Allergic Inflammation That Is Negatively Correlated with Eo Activation

The data of Figure 1-3 implicate the FGN amounts may be lower in the airway tissues of mice with allergic inflammation. To test this, we developed a mouse model of airway allergy with OVA as the specific antigen. The mice showed signs of allergic response in the airway tissues, including the airway hyperresponsiveness (Fig. 4a), high serum levels of OVA-specific IgE, OVAspecific IgG (Fig. $4 \mathrm{~b}-\mathrm{c}$ ), and high levels of MBP in BALF (Fig. 4d). The FGN amounts in the airway tissues were significantly lower in the sensitized group than the NC group (Fig. 4e). The FGN amounts in the lung tissues were negatively correlated with the MBP levels in BALF (Fig. $4 \mathrm{f}-\mathrm{g}$ ). The results suggest that the reduction of FGN amounts in the airway tissues may be associated with the Eo activation in the mice with allergic airway inflammation.

\section{Chymase Physically Contacts FGN to Speed up Its \\ Degradation}

It is known that the chymase levels in the airway tissues are higher in subjects with airway allergy [22]. Chymase can degrade other proteins [5]. We wondered if chymase could interact with FGN in the airway tissues. To this end, we collected the lung tissues; protein extracts were pre- 

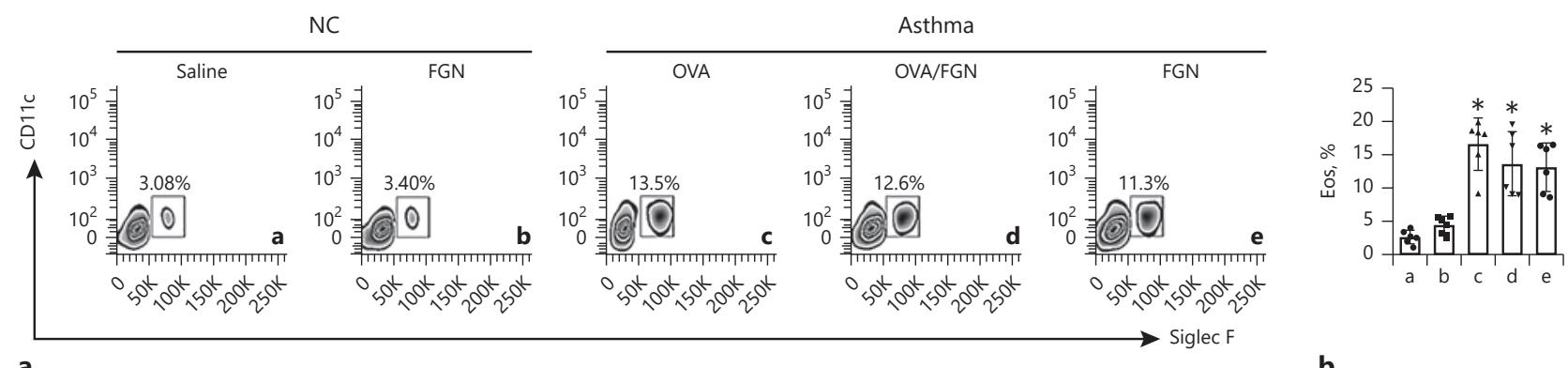

a
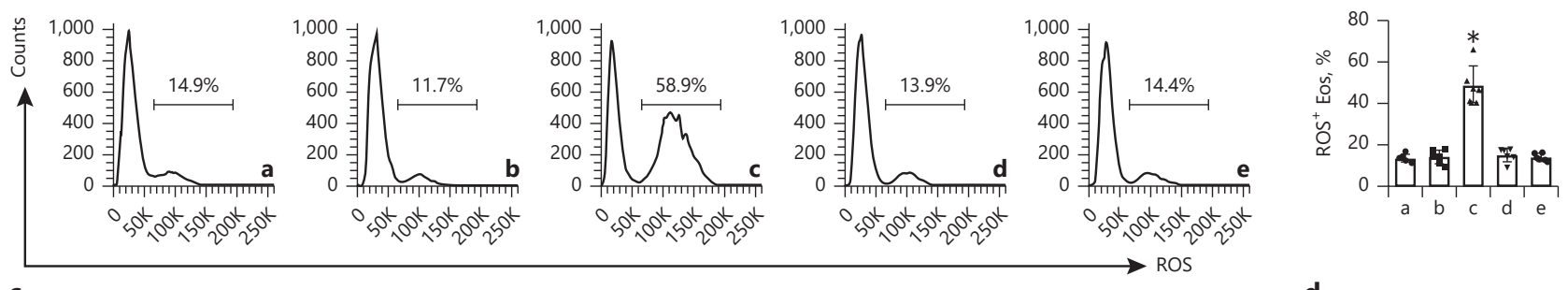

d
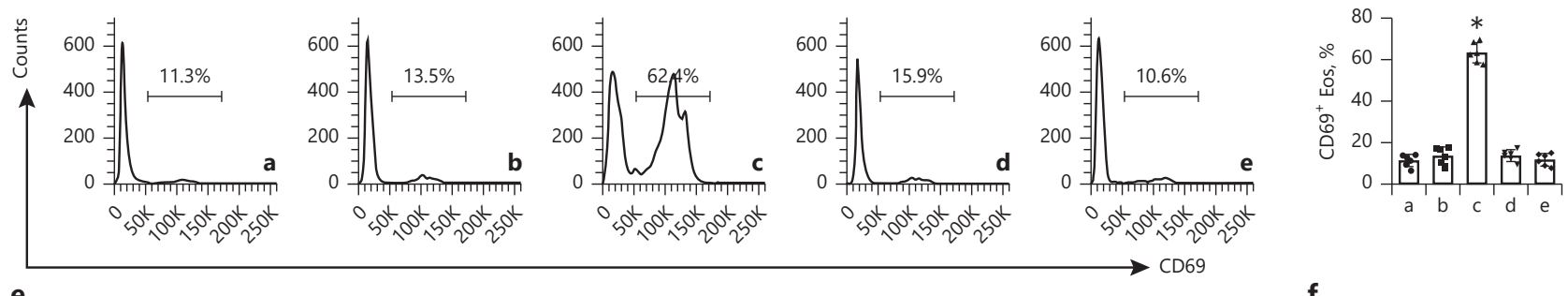

$\mathbf{f}$


Fig. 3. FGN attenuates the specific antigen-induced oxidative stress in Eos. a, d The single cells were isolated from the lung tissues of NC mice and asthma mice. The cells were analyzed by FCS. a, b Gated FCS plots show Eo frequency, and bars show summarized Eo counts in the isolated lung single cells. c, d Gated FCS histograms show ROS $^{+}$Eo frequency, and bars show summarized ROS $^{+}$Eo counts in the gated Eos of a. e, $\mathbf{f}$ Gated FCS histograms show $\mathrm{CD} 69^{+}$Eo frequency, and bars show summarized $\mathrm{CD} 69^{+}$Eos in the gated Eos of a. g, h CD69 $9^{+}$Eos (the activated Eos) were isolated from the lung single cells and analyzed by RT-qPCR and Western blotting. Bars show SOD mRNA levels and immunoblots show SOD protein levels in the activated Eos. i Bars show MBP levels in BALF. j, k Scatter dot plots show positive correlation between BALF MBP levels and $\mathrm{ROS}^{+}$Eos, and negative correlation between BALF MBP levels and SOD in Eos (by Pearson correlation coefficient test). The data of bars are presented as mean \pm SEM. ${ }^{*} p<0.01$ (ANOVA followed by Dunnett's test), compared with group a. Each group consists of 6 mice. The data of $\mathbf{a}, \mathbf{c}, \mathbf{e}, \mathbf{h}$ are from 1 experiment that represents 6 experiments. Each dot in bars presents data obtained from 1 sample. EO, eosinophil; MBP, major basic protein; BALF, bronchoalveolar lavage fluid; FGN, flagellin; FCS, flow cytometry; OVA, ovalbumin; RQ, relative quantification; NC, naive control; ROS, reactive oxygen species; RT-qPCR, real-time quantitative PCR. 


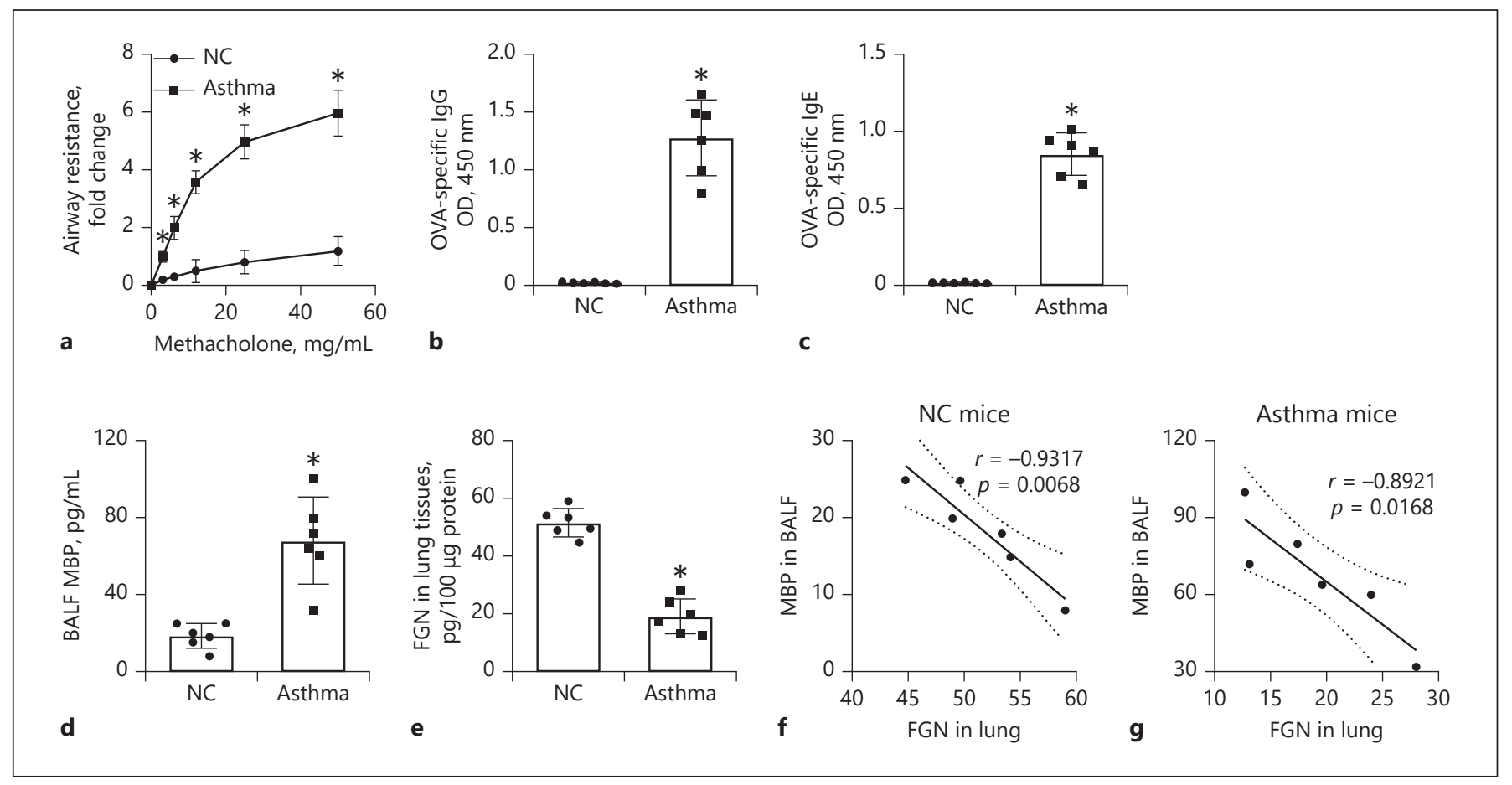

Fig. 4. FGN in the lung is associated with Eo activation. An airway allergy mouse model was developed. a Curves show airway resistance. b, c Bars show serum levels of OVA-specific IgG and OVAspecific IgE. d Bars show MBP levels in BALF. e Bars show FGN levels in the lung tissues. $\mathbf{f}, \mathbf{g}$ Scatter dot plots show correlation between MBP in BALF and FGN in the lung tissues. The data of curve (a) and bars (b-e) are presented in mean \pm SEM. ${ }^{*} p<0.01$,

pared. Chymase and FGN were detected in the protein extracts; the chymase levels were higher in the asthma group than the NC group (Fig. 5a); the FGN levels were lower in the asthma group than that in the $\mathrm{NC}$ group (Fig. 5b). Negative correlation was detected between the data of chymase and FGN in the lung tissues (Fig. 5c-d). The protein extracts were analyzed by co-IP. The results showed that a complex of chymase and FGN was precipitated by either anti-chymase or anti-FGN Ab (Fig. 5e-f). To elucidate if chymase can facilitate the FGN degradation, the Western blotting membrane of chymase/FGN complexes was processed with the "peel-re-blotting" procedures. Ubiquitin was colocalized with FGN in the membrane (Fig. 5e-f) that suggests the FGN degradation [23] by contacting chymase. To verify this, we mixed recombinant chymase and FGN that resulted in the FGN degradation in a chymase concentration-dependent manner (Fig. 5g). The results indicate that chymase, one of the mast cell-derived mediators, causes FGN degradation in the airway tissues. compared with dose 0 (a; ANOVA followed by Dunnett's test) or the NC group (b-e, the Student $t$ test). Statistical method of $\mathbf{f}-\mathbf{g}$, Pearson correlation coefficient test. Each group consists of 6 mice. Each sample was tested in triplicate. Each dot in bars presents data obtained from 1 sample. EO, eosinophil; MBP, major basic protein; BALF, bronchoalveolar lavage fluid; FGN, flagellin; OVA, ovalbumin; NC, naive control.

\section{Administration of FGN Stabilizes Eos in the Lung \\ Tissues of Mice with Airway Allergy}

The above data indicate that FGN can stabilize Eos. Since Eos are the canonical effector inflammatory cells in the airway allergy [24], we inferred that the administration of FGN might attenuate the allergic response in the airways. To this end, we developed an airway allergy mouse model with OVA as the specific antigen. After challenging with OVA, the mice showed asthma-like symptoms, including airway hyper responsiveness, increase in serum specific IgE, specific IgG, IL-4, IL-5, IL13, the higher levels of MBP, and high Eo counts in BALF (Fig. 6a-k). The administration of FGN in the course of sensitization efficiently alleviated asthma-like symptoms (Fig. 6a-b), although high levels of specific IgE/IgG and Th2 cytokines were detected in the serum (Fig. 6c-g); however, Eo counts and MBP levels were markedly reduced in BALF of mice treated with FGN (Fig. 6h-j). The results demonstrate that the administration of FGN can alleviate the experimental airway allergy. 


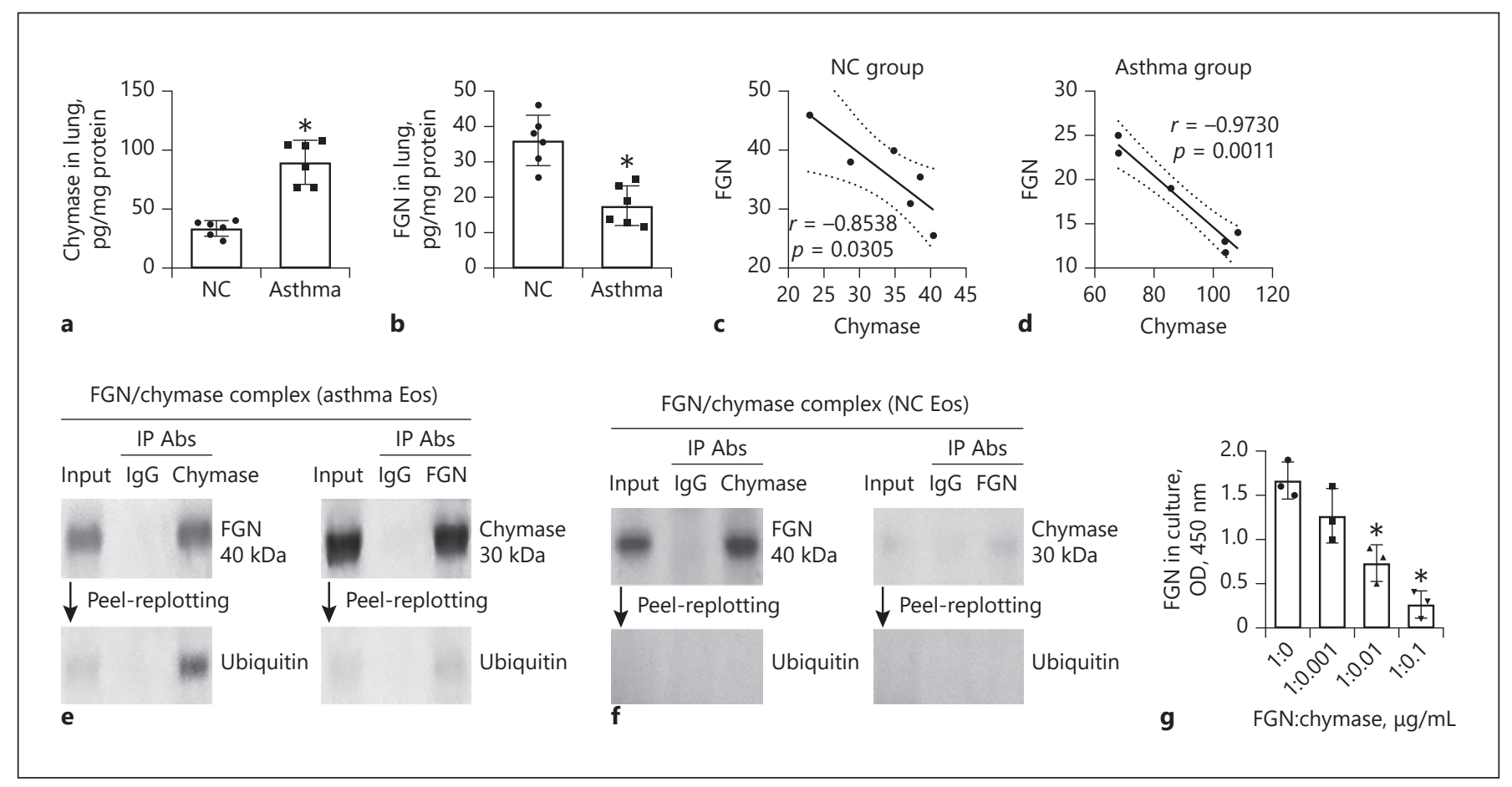

Fig. 5. Assessment of the role of chymase in degrading FGN in the airway tissues. Lung tissues were excised from NC mice $(n=6)$ and asthma mice $(n=6)$. Proteins were extracted from the samples. a, b Bars show the levels of chymase (a) and FGN (b) in the lung tissues. c, d Scatter dot plots show negative correlation between FGN and chymase levels in the lung of NC mice (c) and asthma mice (d). e, f Co-IP results show FGN/chymase complexes in the lung tissues. The lower gel graphs show ubiquitin colocalized in

\section{Discussion}

The present study revealed a previously undescribed phenomenon that FGN contributed to Eo stabilization in the airway tissues. We found that TLR5 KO mice showed the Eo spontaneous activation and release of mediators that was verified by a cell culture experiment, in which Eos spontaneously released mediators in the absence of FGN. Upon specific antigen challenge, oxidative stress was induced in Eos of the airway tissues that activated Eos. FGN increased the SOD expression to inhibit oxidative stress in Eos and thus, stabilized Eos. The study also revealed that the high chymase levels in the tissues with allergic lesions were the causative factor of the low FGN levels. The administration of FGN efficiently alleviated experimental asthma.

We observed the spontaneous Eo activation in the airway tissues of TLR5 KO mice. To employ TLR5 KO mice is to mimic an environment of the FGN derivation. As
FGN bots. $\mathbf{g}$ Bars show FGN levels after mixing with chymase (at gradient concentrations) in the culture. The data of bars are presented as mean \pm SEM. ${ }^{*} p<0.01$, compared the NC group (a, b; the Student $t$ test) or the "1:0" group (g; ANOVA followed by Dunnett's test). The data of $\mathbf{e}, \mathbf{f}$ are from 1 experiment that represents 3 experiments. Each dot in bars presents data obtained from 1 sample. NC, naive control; FGN, flagellin; IP, immunoprecipitation; Abs, antibodies.

high serum levels of Eo mediator were detected in the serum as well as in BALF, the data implicate that FGN is required in maintaining the Eo stabilization. The previous studies showed that about 10\% TLR5 KO mice had colitis [25]; this may be attributed to the hyper-expression of hematopoietic pro-inflammatory cytokines [26]. TLR5 $\mathrm{KO}$ mice are also prone to suffer microbiota-related metabolic syndrome [27]. These findings suggest that TLR5 signals are involved in the immune regulation that supports the proposal that TLR5 signal is required in the maintenance of Eo stabilization.

The data show that, upon exposure to specific antigens, oxidative stress was induced in Eos in the airway tissues of mice with airway allergy. Oxidative stress can be induced in the cells by multiple factors [28]. The allergic response is one of them [29]. Oxidative stress in Eos has also been reported. The published data show that the Eo recruitment is positively correlated with the oxidative stress states in the local tissues [30]. Surfactant protein D, 


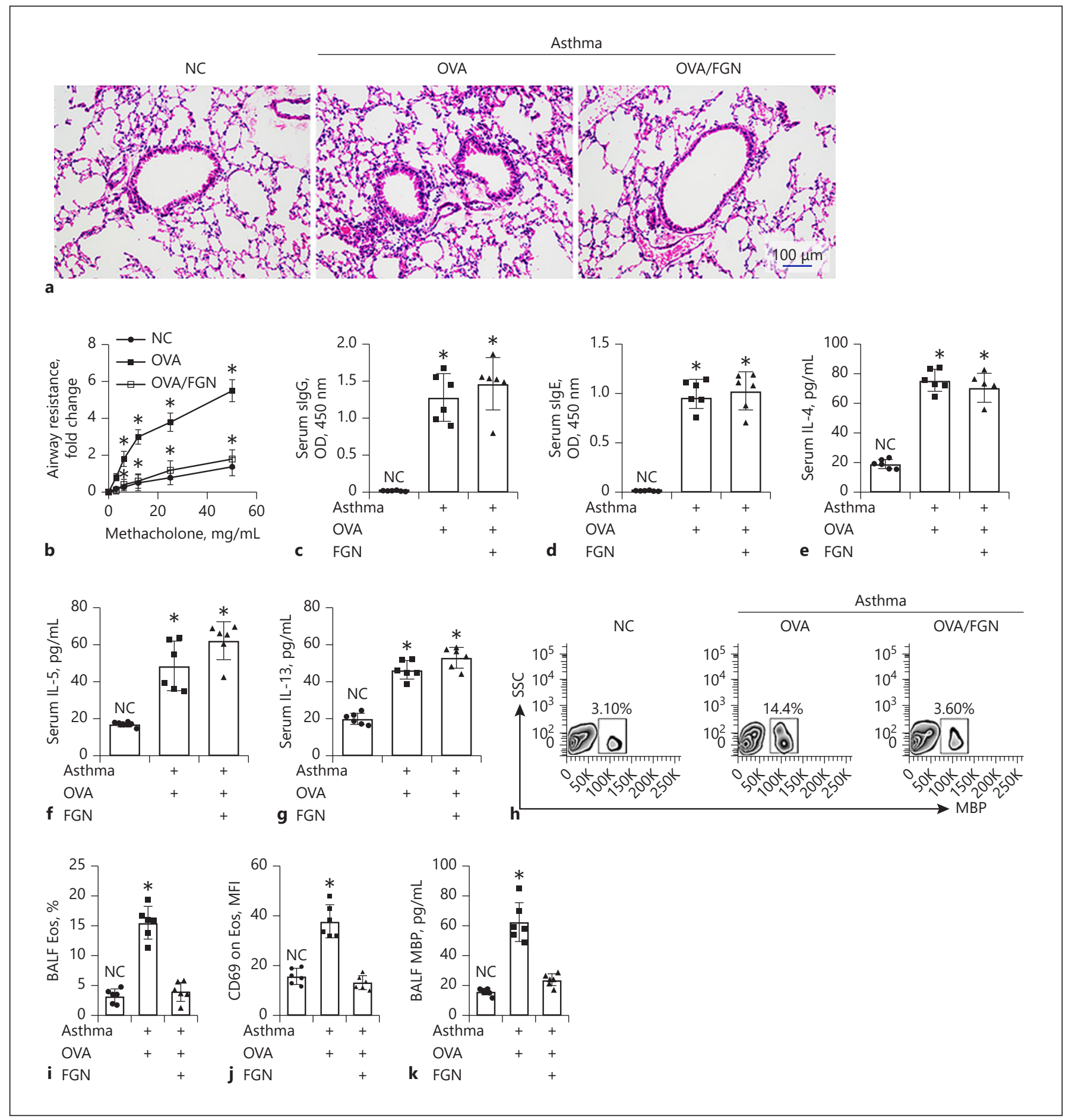

Fig. 6. Administration of FGN alleviates experimental airway allergy. An asthma mouse model was developed with OVA as the specific antigen. a Representative lung tissue histology images (original magnification, $\times 200$ ). b The curves show the airway resistance. $\mathbf{c}-\mathbf{g}$ The bars show serum levels of specific IgG, specific IgE, IL-4, IL-5, and IL-13. $\mathbf{h}$ The gated FCS plots show Eo frequency in BALF. $\mathbf{i}$ The bars show summarized Eo counts in BALF. $\mathbf{j}$ The bars show CD69 MFI on BALF Eos. $\mathbf{k}$ The bars show BALF MBP levels. The data of bars are presented as mean \pm SEM. ${ }^{*} p<0.01$ (ANOVA followed by Dunnett's test), compared with the NC group. Each group consists of 6 mice. The data of $\mathbf{h}$ are from 1 experiment that represents 6 experiments. Each dot in bars presents data obtained from 1 sample. EO, eosinophil; MBP, major basic protein; BALF, bronchoalveolar lavage fluid; FGN, flagellin; OVA, ovalbumin; NC, naive control. 
a C-type lectin, can increase oxidative stress in Eos and activate Eos [31]. The present data have added novel information to this study area. Exposure to specific antigens can induce oxidative stress in Eos in the tissues with allergic lesions. This is supported by the previous reports in which the exposure to specific antigens can activate Eos by binding the specific IgG/Fc $\gamma$ RI or/and Fc $\gamma$ RIII on the surface of Eos $[9,20]$.

The data show that exposure to FGN can attenuate oxidative stress in Eos. This is a novel finding. As a major component of the bacterial flagella, FGN distributes extensively in the human living environment as well as inside the body cavities, such as in the intestine [32]. FGN has a unique receptor, TLR5. By ligating TLR5, FGN is able to regulate cell activities [33]. The present data show that the interaction of FGN/TLR5 promotes the SOD expression. Since SOD is one of the major antioxidants in the body, it is logical that FGN can counteract the oxidative stress-related Eo activation.

We also found that the FGN levels were lower in the airway tissues of mice with allergic airway inflammation than NC. Although there has been no published data showing the causes of FGN amount reduction in the airway tissues, we found that chymase, one of the mast cellderived proteases, could induce the FGN degradation. For example, the previous studies indicate that chymase can degrade fibrinogen, fibrin, and intracellular procollagen I $[5,34]$. The present data provide evidence that chymase can cause low FGN levels in the airway tissues by promoting FGN degradation.

The data show that administration of FGN can efficiently alleviate the asthma-like symptoms in mice. The underlying mechanism of the therapeutic effects is based on stabilizing Eos in the airway tissues. We found that although markedly higher serum specific IgE/IgG levels and high MBP levels in BALF in mice with airway allergy, no appreciable clinical symptoms were evoked, the BALF Eo counts, Eo activation, and MBP levels were not increased in the mice treated with FGN/OVA. The results indicate that FGN can stabilize Eos against the specific antigen challenge and thus prevent the allergic response. The results also mirror the importance of Eos in the pathogenesis of allergic disorders.

This work focused on the role of FGN in stabilizing Eos, while there are several questions about FGN that were not studied by the present study. For example, if FGN coming from other than generic flagella of bacteria in the human living environment also can be found in the lung? What is the functional difference in FGN from the lungs of asthmatic subjects versus healthy subjects? Al- though these questions do not fall in the scope of the present study, it may be of significance in immunological studies and need to be investigated further in the future studies.

In summary, the present data show that FGN has the effects on stabilizing Eos in the airway tissues. The administration of FGN has the effects on alleviating airway allergic inflammation that has the therapeutic potential to be employed in the treatment of airway allergy.

\section{Statement of Ethics}

The animal experimental procedures were approved by the Animal Ethical Committee at Southern Medical University (SMUAEC2019022).

\section{Conflict of Interest Statement}

The authors have no conflicts of interest to declare.

\section{Funding Sources}

This study was supported by grants of the National Nature and Science Foundation of China (32090052, 81870706, 31570932, U1801286, 31000713, and 31700805), Guangdong Provincial Key Laboratory of Regional Immunity and Diseases (2019B030301009), Shenzhen Nanshan District Oversea Research Personnel Initiative Group Fund (LHTD20180007), and Shenzhen Science, Technology, and Innovation Committee (KQTD20170331145453160 and KQJSCX20180328095619081).

\section{Author Contributions}

X.Q.L., J.L., L.H.M., G.Y., F.M., and Y.N. performed the experiments, analyzed data, and reviewed the manuscript. P.C.Y. and D.B.L. organized the study and supervised the experiments. P.C.Y. designed the project and prepared the manuscript.

References

1 Rothenberg ME, Hogan SP. The eosinophil. Annu Rev Immunol. 2006;24:147-74.

2 Weller PF, Spencer LA. Functions of tissueresident eosinophils. Nat Rev Immunol. 2017; 17:746-60

3 Fulkerson PC, Rothenberg ME. Eosinophil development, disease involvement, and therapeutic suppression. Adv Immunol. 2018; 138:1-34.

4 González-de-Olano D, Álvarez-Twose I. Mast cells as key players in allergy and inflammation. J Investig Allergol Clin Immunol. 2018; 28:365-78. 
5 Lipitsä T, Siiskonen H, Naukkarinen A, Harvima IT. Mast cell chymase degrades fibrinogen and fibrin. Br J Dermatol. 2019;181:296303.

6 Nanagas VC, Kovalszki A. Gastrointestinal manifestations of hypereosinophilic syndromes and mast cell disorders: a comprehensive review. Clin Rev Allergy Immunol. 2019;57:194-212.

7 Johansson MW. Eosinophil activation status in separate compartments and association with asthma. Front Med. 2017;4:75

8 Hellmark T, Ohlsson S, Pettersson Å, Hansson M, Johansson ÅCM. Eosinophils in antineutrophil cytoplasmic antibody associated vasculitis. BMC Rheumatol. 2019;3:9.

9 An YF, Suo LM, Xue JM, Han HY, Yang G, Liu JQ, et al. Role of Fc $\gamma$ RI in antigen-dependent eosinophil activation in patients with allergic rhinitis. Am J Rhinol Allergy. 2020: 1945892420936587.

10 Evans H, Killoran KE, Mitre BK, Morris CP, Kim SY, Mitre E. Ten weeks of infection with a tissue-invasive helminth protects against local immune complex-mediated inflammation, but not cutaneous type i hypersensitivity, in previously sensitized mice. J Immunol. 2015;195:2973-84.

11 Wong CK, Cheung PF, Ip WK, Lam CW. Intracellular signaling mechanisms regulating toll-like receptor-mediated activation of eosinophils. Am J Respir Cell Mol Biol. 2007;37: 85-96.

12 Hajam IA, Dar PA, Shahnawaz I, Jaume JC, Lee JH. Bacterial flagellin-a potent immunomodulatory agent. Exp Mol Med. 2017;49: e373.

13 Tran HQ, Ley RE, Gewirtz AT, Chassaing B Flagellin-elicited adaptive immunity suppresses flagellated microbiota and vaccinates against chronic inflammatory diseases. Nat Commun. 2019;10:5650.

14 Vijayan A, Rumbo M, Carnoy C, Sirard JC. Compartmentalized antimicrobial defenses in response to flagellin. Trends Microbiol. 2018;26:423-35.

15 Wilson RH, Maruoka S, Whitehead GS, Foley JF, Flake GP, Sever ML, et al. The Toll-like receptor 5 ligand flagellin promotes asthma by priming allergic responses to indoor allergens. Nat Med. 2012;18:1705-10.

16 Kim EH, Kim JH, Samivel R, Bae JS, Chung YJ, Chung PS, et al. Intralymphatic treatment of flagellin-ovalbumin mixture reduced allergic inflammation in murine model of allergic rhinitis. Allergy. 2016;71:629-39.

17 Kelly EA, Esnault S, Liu LY, Evans MD, Johansson MW, Mathur S, et al. Mepolizumab attenuates airway eosinophil numbers, but not their functional phenotype, in asthma. Am J Respir Crit Care Med. 2017;196:138595.

18 Padigel UM, Lee JJ, Nolan TJ, Schad GA, Abraham D. Eosinophils can function as antigen-presenting cells to induce primary and secondary immune responses to Strongyloides stercoralis. Infect Immun. 2006;74:32328

19 Lin J, Huang N, Li J, Liu X, Xiong Q, Hu C, et al. Cross-reactive antibodies against dust mite-derived enolase induce neutrophilic airway inflammation. Eur Respir J. 2020.

20 Davoine F, Labonté I, Ferland C, Mazer B, Chakir J, Laviolette M. Role and modulation of CD16 expression on eosinophils by cytokines and immune complexes. Int Arch $\mathrm{Al}$ lergy Immunol. 2004;134:165-72.

21 de Groot LES, Sabogal Piñeros YS, Bal SM, van de Pol MA, Hamann J, Sterk PJ, et al. Do eosinophils contribute to oxidative stress in mild asthma? Clin Exp Allergy. 2019;49:92931.

22 Zoltowska AM, Lei Y, Fuchs B, Rask C, Adner $\mathrm{M}$, Nilsson GP. The interleukin-33 receptor ST2 is important for the development of peripheral airway hyperresponsiveness and inflammation in a house dust mite mouse model of asthma. Clin Exp Allergy. 2016;46:47990.

23 Varshavsky A. The ubiquitin system, autophagy, and regulated protein degradation. Annu Rev Biochem. 2017;86:123-8.

24 Busse W, Chupp G, Nagase H, Albers FC, Doyle S, Shen Q, et al. Anti-IL-5 treatments in patients with severe asthma by blood eosinophil thresholds: indirect treatment compari- son. J Allergy Clin Immunol. 2019;143:190e20.

25 Singh V, Yeoh BS, Carvalho F, Gewirtz AT, Vijay-Kumar M. Proneness of TLR5 deficient mice to develop colitis is microbiota dependent. Gut Microbes. 2015;6:279-83.

26 Vijay-Kumar M, Sanders CJ, Taylor RT, Kumar A, Aitken JD, Sitaraman SV, et al. Deletion of TLR5 results in spontaneous colitis in mice. J Clin Invest. 2007;117:3909-21.

27 Singh V, Chassaing B, Zhang L, San Yeoh B, Xiao X, Kumar M, et al. Microbiota-dependent hepatic lipogenesis mediated by stearoyl coa desaturase 1 (SCD1) promotes metabolic syndrome in TLR5-deficient mice. Cell Metab. 2015;22:983-96.

28 Dandekar A, Mendez R, Zhang K. Cross talk between ER stress, oxidative stress, and inflammation in health and disease. Methods Mol Biol. 2015;1292:205-14.

29 Mishra V, Banga J, Silveyra P. Oxidative stress and cellular pathways of asthma and inflammation: therapeutic strategies and pharmacological targets. Pharmacol Ther. 2018;181:169-82.

30 Hanazawa T, Antuni JD, Kharitonov SA Barnes PJ. Intranasal administration of eotaxin increases nasal eosinophils and nitric oxide in patients with allergic rhinitis. J Allergy Clin Immunol. 2000;105:58-64.

31 Mahajan L, Gautam P, Dodagatta-Marri E, Madan T, Kishore U. Surfactant protein SP-D modulates activity of immune cells: proteomic profiling of its interaction with eosinophilic cells. Expert Rev Proteomics. 2014;11: 355-69.

32 Gewirtz AT. Flag in the crossroads: flagellin modulates innate and adaptive immunity. Curr Opin Gastroenterol. 2006;22:8-12.

33 McNamara N, Gallup M, Sucher A, Maltseva I, McKemy D, Basbaum C. AsialoGM1 and TLR5 cooperate in flagellin-induced nucleotide signaling to activate Erk1/2. Am J Respir Cell Mol Biol. 2006;34:653-60.

34 Fu L, Wei CC, Powell PC, Bradley WE, Ahmad S, Ferrario CM, et al. Increased fibroblast chymase production mediates procollagen autophagic digestion in volume overload. J Mol Cell Cardiol. 2016;92:1-9. 